\title{
BROUWER DEGREE, EQUIVARIANT MAPS AND TENSOR POWERS
}

\author{
Z. BALANOV*, W. KRAWCEWICZ** AND A. KUSHKULEY
}

\begin{abstract}
A construction of equivariant maps based on factorization through symmetric powers of a faithful representation is presented together with several examples of related equivariant maps. Applications to differential equations are also discussed.
\end{abstract}

\section{IntRoduCtion}

Let $G$ be a compact Lie group and $V, W$ two orthogonal $G$-representations of the same dimensions. We denote by $S(V)$ the unit sphere in $V$.

The following question was raised by J.F. Adams in 1963 (cf. [1,9]) whether there exists a G-equivariant map $f: S(V) \rightarrow S(W)$ of a given degree $\operatorname{deg}(f)=k$. From the point of view of applications in analysis, the case $k \neq 0$ has particular importance. For a finite $p$-group $G$, a complete answer to this question was given by M. Atiyah and D. Tall (cf. [2]). To be more specific, a map $f$ with degree $\operatorname{deg}(f) \not \equiv 0(\bmod p)$ exists if and only if the irreducible components of $V$ and $W$ are conjugate in pairs by (possibly different) elements of the Galois group "associated" to $G$.

In the general case of a compact Lie group $G$, the congruence relations between possible values of degrees of equivariant maps are described in the following result:

1991 Mathematics Subject Classification. Primary: 58C30; Secondary: 47H11, 55M25, 55M35, 57S15.

Key words and phrases. Brouwer degree, equivariant maps, tensor powers.

* Research supported by the Alexander von Humboldt Foundation and a grant from PIMS.

** Research supported by NSERC (Canada) and the Alexander von Humboldt Foundation.

Received: March 2, 1998.

(C) 1996 Mancorp Publishing, Inc. 
Theorem 1.1. Let $f_{1}, f_{2}: S(V) \rightarrow S(W)$ be two $G$-equivariant maps. Then

$$
\operatorname{deg}\left(f_{1}\right) \equiv \operatorname{deg}\left(f_{2}\right) \quad(\bmod \alpha),
$$

where $\alpha=\operatorname{gcd}\left(\chi\left(G / H_{1}\right), \ldots, \chi\left(G / H_{n}\right)\right), \chi(\cdot)$ stands for the Euler characteristic, and $\left\{\left(H_{1}\right), \ldots,\left(H_{n}\right)\right\}$ are all the orbit types in $S(V)$ satisfying

(i) $H_{i}$ is of maximal rank;

(ii) $\operatorname{dim} V^{H_{i}}=\operatorname{dim} W^{H_{i}}$;

(iii) there exists an $H_{i}$-equivariant map between "complementing" spheres to $V^{H_{i}}$ and $W^{H_{i}}$, respectively ${ }^{1}$.

The proof as well as the more general formulation of Theorem 1.1 can be found in $[8]$. We also refer to $[5,6]$ for the discussion and related results in the case where $G$ is an abelian group.

Evidently, in order to use this result, one has to answer the question if there exists an equivariant map $f_{1}$ with degree "simple" to calculate for which the congruence relation (1.1) would imply the nontriviality of $\operatorname{deg}\left(f_{2}\right)$.

On the other hand, computations of important invariants of variational problems with symmetries (such as genus, $G$-category, etc.) lead to the problem of (non)existence of equivariant map $f: S(V) \rightarrow S(W)$ with $\operatorname{dim} V>\operatorname{dim} W$ (we refer to [3] for a general discussion of this question and several elegant examples). We would like to point out that this problem is also closely related to the verification of condition (iii) of Theorem 1.1 (cf. [8] and $[5,6]$ ).

This paper is intended to reveal common features behind certain examples of equivariant maps related to the above issues. Actually, we would like to describe an approach to explicit construction of equivariant maps by exploiting classic methods of the representation/invariant theory (for all relevant information, we refer to $[4,13])$.

In what follows we will always assume that all the considered complex (resp. real) representation are unitary (resp. orthogonal). A map $f: V \rightarrow$ $W$ is said to be nonsingular if $f(x) \neq 0$ for $x \neq 0$ (cf. [7]).

The authors are grateful to S. Brodsky for his important suggestions and inspiring expertise. We would like also to thank T. Bartsch, J. Ize and D. Verma for fruitful discussions and/or comments.

\section{EXAMPLES}

We denote by $\mathbb{H}=\mathbb{C}^{2}=\left\{z_{1}+j z_{2} ; z_{1}, z_{2} \in \mathbb{C}\right\}$ the algebra of quaternions, where the multiplication satisfies the standard properties $j^{2}=-1$, $j i=-i j$. We also identify the cyclic group $\mathbb{Z}_{n}$ with $\left\{\gamma \in \mathbb{C} ; \gamma^{n}=1\right\}$. The group $\mathbf{Q}_{8}$ of quaternionic units is defined as $\{ \pm 1, \pm i, \pm j, \pm j i\} \subset \mathbb{H}$, that is $\mathbf{Q}_{8}=\mathbb{Z}_{4} \cup j \mathbb{Z}_{4}$. For the Klein group $\mathbb{Z}_{2} \times \mathbb{Z}_{2}$ we use the notation:

\footnotetext{
${ }^{1}$ We refer to [8] for the precise formulation of this rather technical condition which is related to the "secondary obstructions" in the equivariant context (cf. $[5,6]$ )
} 
$1=(1,1),-1=(-1,-1), \quad \kappa=(1,-1),-\kappa=(-1,1)$. Notice, $\mathbb{Z}_{2}$ and $\mathbb{Z}_{4}$ are normal in $\mathbf{Q}_{8}$. The homomorphisms $\alpha: \mathbf{Q}_{8} \rightarrow \mathbf{Q}_{8} / \mathbb{Z}_{2} \simeq \mathbb{Z}_{2} \times \mathbb{Z}_{2}$, $\alpha(z)=z^{2}$ and $\alpha(j z)=\kappa z^{2}$, where $z \in\{ \pm 1, \pm i\}$, and $\beta: \mathbf{Q}_{8} \rightarrow \mathbf{Q}_{8} / \mathbb{Z}_{4} \simeq \mathbb{Z}_{2}$, $\beta\left(z_{1}+j z_{2}\right)=z_{1}^{2}-z_{2}^{2}$, are well defined quotient projections.

Clearly, $\mathbf{Q}_{8}$ acts on $\mathbb{H}$ by the quaternionic multiplication and we denote by $\left(\mathbb{H}, \mathbf{Q}_{8}\right)$ the corresponding $\mathbf{Q}_{8}$-representation. The $\mathbb{Z}_{2} \times \mathbb{Z}_{2}$-action on $\mathbb{C}^{2}$ given by

$$
\left( \pm 1,\left(z_{1}, z_{2}\right)\right) \mapsto\left( \pm z_{1}, \pm z_{2}\right), \quad\left( \pm \kappa,\left(z_{1}, z_{2}\right)\right) \mapsto\left(\mp z_{1}, \pm z_{2}\right),
$$

induces via $\alpha$ a (complex) $\mathbf{Q}_{8}$-representation which we will denote by $\left(\mathbb{C}^{2}, \mathbf{Q}_{8} / \mathbb{Z}_{2}\right)$.

A $\mathbb{Z}_{2} \times \mathbb{Z}_{2}$-action on $\mathbb{R}^{3}=\mathbb{C} \oplus \mathbb{R}$ is defined by

$$
( \pm 1,(z, t)) \mapsto( \pm z, t), \quad( \pm \kappa,(z, t)) \mapsto(\mp \bar{z}, \mp t)
$$

and the corresponding (real) $\mathbf{Q}_{8}$-representation will be denoted by $\left(\mathbb{R}^{3}, \mathbf{Q}_{8} / \mathbb{Z}_{2}\right)$. Finally, the antipodal $\mathbb{Z}_{2}$-action on $\mathbb{R}^{3}$ induces via $\beta$ a (real) Q8-representation $\left(\mathbb{R}^{3}, \mathbf{Q}_{8} / \mathbb{Z}_{4}\right)$.

The following examples of equivariant maps are the starting point for our discussion.

Example 2.1. We define a map $\tilde{f}: \mathbb{H} \rightarrow \mathbb{C}^{2}$ by

$$
\tilde{f}\left(z_{1}+j z_{2}\right):=\left(z_{1}^{2}+z_{2}^{2}, z_{1}^{2}-z_{2}^{2}\right), \quad z_{1}+j z_{2} \in \mathbb{H} .
$$

By direct verification $\tilde{f}$ is a $\mathbf{Q}_{8}$-equivariant nonsingular map from $\left(\mathbb{H}, \mathbf{Q}_{8}\right)$ to $\left(\mathbb{C}^{2}, \mathbf{Q}_{8} / \mathbb{Z}_{2}\right)$. Consequently, we have a $\mathbf{Q}_{8}$-equivariant map $f: S(\mathbb{H}) \rightarrow$ $S\left(\mathbb{C}^{2}\right)$, given by $f(x):=\tilde{f}(x) /\|\tilde{f}(x)\|, x \in S(\mathbb{H})$. Clearly, $\operatorname{deg} f=4$, hence Theorem 1.1 yields

Proposition 2.2. For any $\mathbf{Q}_{8}$-equivariant map $\psi: S(\mathbb{H}) \rightarrow S\left(\mathbb{C}^{2}\right)$ one has $\operatorname{deg} \psi \equiv 4(\bmod 8)$. In particular, $\operatorname{deg} \psi$ is different from zero.

Remark 2.3. Notice that the statement of Proposition 2.2 does not follow from the well known results for abelian groups (see, for example, $[5,6]$ ). Indeed, there are only two abelian subgroups in $\mathbf{Q}_{8}$, namely $\mathbb{Z}_{2}$ and $\mathbb{Z}_{4}$ for which the "restriction" of the actions leads to the congruencies $\operatorname{deg} \psi \equiv 4$ $(\bmod 2)$ and $\operatorname{deg} \psi \equiv 4(\bmod 4)$, respectively. This information is insufficient to conclude that $\operatorname{deg} \psi \neq 0$, by the same token, the situation described in Example 2.1 is of non-abelian "nature".

Remark 2.4. Observe also that Example 2.1 is compatible with the above mentioned Atiyah-Tall result (cf. [2]). For, since $\left(\mathbb{H}, \mathbf{Q}_{8}\right)$ and $\left(\mathbb{C}^{2}, \mathbf{Q}_{8} / \mathbb{Z}_{2}\right)$ are two non-conjugate representations (by an element of the Galois group) of the 2-group $\mathbf{Q}_{8}$, every equivariant map $\psi: S(\mathbb{H}) \rightarrow S\left(\mathbb{C}^{2}\right)$ satisfies $\operatorname{deg} \psi \equiv 0$ $(\bmod 2)$. Notice that in Example $2.1 \operatorname{deg} f=4=2^{2} \equiv 0(\bmod 2)$. 
Example 2.5. We consider the map $g: \mathbb{H} \rightarrow \mathbb{C} \oplus \mathbb{R} \simeq \mathbb{R}^{3}$ given by

$$
g\left(z_{1}+j z_{2}\right)=\left(z_{1}^{2}-\bar{z}_{2}^{2}, \Re e\left(z_{1} z_{2}\right)\right), \quad z_{1}+j z_{2} \in \mathbb{H} .
$$

Clearly, $g$ is a $\mathbf{Q}_{8}$-equivariant nonsingular map from $\left(\mathbb{H}, \mathbf{Q}_{8}\right)$ to $\left(\mathbb{R}^{3}, \mathbf{Q}_{8} / \mathbb{Z}_{2}\right)$.

Remark 2.6. The map $g$ was originally constructed by T. Bartsch (cf. [3], Example 3.21) for the representations $\left(\mathbb{H}, \mathbb{Z}_{4}\right)$ and $\left(\mathbb{R}^{3}, \mathbb{Z}_{4} / \mathbb{Z}_{2}\right)$, where $\mathbb{Z}_{4} \simeq$ $\{1,-1, j,-j\} \subset \mathbf{Q}_{8}$ acts on $\mathbb{H}$ by the quaternionic multiplication (which is the standard action of $\mathbb{Z}_{4}$ on $\mathbb{C}^{2} \simeq \mathbb{H}$ ), and $\mathbb{Z}_{4} / \mathbb{Z}_{2}$ acts antipodally on $\mathbb{R}^{3}$. For more information on the connection between decreasing dimension equivariant maps and estimates of certain topological invariants (such as genus, $G$-category, etc.) related to variational problems with symmetries, we refer the interested reader to the book [3] (see also the references therein). By direct computation one can verify that the Hopf invariant of $g$ is equal to -1 .

Remark 2.7. We should also mention Example 4.1 from [6] (which was partially inspired by Bartsch's example) describing possible values of Brouwer degrees of equivariant maps "generic" in a certain sense. The problem of existence of decreasing dimension equivariant maps is also related to the "secondary obstructions" in the equivariant extension context (see $[5,6,8]$ for more details).

Example 2.8. It is easy to verify that the map $h: \mathbb{H} \rightarrow \mathbb{C} \oplus \mathbb{R}=\mathbb{R}^{3}$, given by

$$
h\left(z_{1}+j z_{2}\right)=\left(z_{1}^{2}-z_{2}^{2}, \Re e\left(\bar{z}_{1} z_{2}\right)\right),
$$

is a $\mathbf{Q}_{8}$-equivariant nonsingular map from $\left(\mathbb{H}, \mathbf{Q}_{8}\right)$ to $\left(\mathbb{R}^{3}, \mathbf{Q}_{8} / \mathbb{Z}_{4}\right)$.

Example 2.8. Let $\mathbb{H}$ be a $\mathbf{Q}_{8 n}$-representation ( $n \geq 2$ ), where $\mathbf{Q}_{8 n}=\mathbb{Z}_{4 n} \cup$ $j \mathbb{Z}_{2 n} \subset \mathbb{H}$ acts on $\mathbb{H}$ by quaternionic multiplication. If $\mathbb{Z}_{2}$ acts antipodally on $\mathbb{R}^{3}$, the natural homomorphism $\mathbf{Q}_{8 n} \rightarrow \mathbf{Q}_{8 n} / \mathbb{Z}_{4 n} \simeq \mathbb{Z}_{2},\left(z_{1}+j z_{2}\right) \mapsto$ $z_{1}^{2 n}-z_{2}^{2 n}, z_{i} \in \mathbb{Z}_{4 n}, \quad i=1,2$, gives us a $\mathbf{Q}_{8 n}$-representation on $\mathbb{R}^{3}$. Notice that the formula

$$
h\left(z_{1}, z_{2}\right)=\left(z_{1}^{2 n}-z_{2}^{2 n}, \Re e\left(\bar{z}_{1}^{n} z_{2}^{n}\right)\right), \quad z_{1}, z_{2} \in \mathbb{C},
$$

determines evidently a $\mathbf{Q}_{8 n}$-equivariant nonsingular map from $\left(\mathbb{H}, \mathbf{Q}_{8 n}\right)$ to $\left(\mathbb{R}^{3}, \mathbf{Q}_{8 n} / \mathbb{Z}_{4 n}\right)$. The Hopf invariant of $h$ is $n$.

\section{Construction}

Let $V$ be a faithful (complex or real) finite-dimensional representation of a compact Lie group $G$. We are interested in describing $G$-representations $W$ which admit $G$-equivariant maps from $S(V)$ into $S(W)$. It is well known that in the most interesting cases, for any irreducible $G$-representation $U$, 
there exists a tensor power $V^{\otimes k}:=V \underbrace{\otimes \cdots \otimes}_{k \text { times }} V$, with the standard $G$-action, which contains $U$.

Fix $k \geq 2$ and consider the "diagonal" map $\triangle: V \rightarrow V^{\otimes k}, \triangle(v)=$ $v \underbrace{\otimes \cdots \otimes}_{k \text { times }} v, v \in V$. Clearly, in general $\triangle(V)$ is not a linear subspace of $V^{\otimes k}$, however, it is $G$-invariant and the set $\triangle(V)$ spans the symmetric $k$-power $\operatorname{Sym}^{k}(V)$ of the space $V$ (see [4]). Thus $\triangle: V \rightarrow \operatorname{Sym}^{k}(V)$ is a $G$-equivariant map. Let $W \subset \operatorname{Sym}^{k}(V)$ be a subrepresentation and $A: \operatorname{Sym}^{k}(V) \rightarrow W$ a $G$-equivariant linear operator.

We make the following assumption:

(A) $\operatorname{ker} A \cap \triangle(V)=\{0\}$.

As an immediate consequence we obtain

Proposition 3.1. Under the assumption $(A)$ the map $\tilde{f}: V \rightarrow W$, given by $\tilde{f}(v)=A(\triangle(v)), v \in V$, is a $G$-equivariant nonsingular map.

Remark 3.2. Suppose that $V$ is a complex representation and assume that the center $Z(G)$ of the group $G$ is a discrete subgroup of order $k$. Then, clearly, $Z(G)$ acts trivially on $\operatorname{Sym}^{k}(V)$ (this fact in certain cases is also true for real representations). Consequently, every subrepresentation $W \subset \operatorname{Sym}^{k}(V)$ is not equivalent to $V$.

Let us reproduce the equivariant maps from Examples 2.1, 2.5 and 2.8 using the above construction.

Example 3.3. We consider the (complex) representation $\left(\mathbb{H}, \mathbf{Q}_{8}\right)$ and its second complex symmetric power $\operatorname{Sym}_{\mathbb{C}}^{2}(\mathbb{H})$. Notice, the center $\mathbb{Z}_{2}$ of $\mathbf{Q}_{8}$ acts trivially on $\operatorname{Sym}_{\mathbb{C}}^{2}(\mathbb{H})$, i.e. the second symmetric power "kills" the center, hence $\mathbf{Q}_{8}$ acts on $\operatorname{Sym}_{\mathbb{C}}^{2}(\mathbb{H})$ as $\mathbf{Q}_{8} / \mathbb{Z}_{2} \simeq \mathbb{Z}_{2} \times \mathbb{Z}_{2}$. One can easily check that

$$
e_{1}=\frac{1 \otimes 1+j \otimes j}{2}, \quad e_{2}=\frac{1 \otimes 1-j \otimes j}{2}, \quad e_{3}=\frac{j \otimes 1+1 \otimes j}{2},
$$

is an invariant basis for $\operatorname{Sym}_{\mathbb{C}}^{2}(\mathbb{H})$ and

$$
\triangle\left(z_{1}+j z_{2}\right)=e_{1}\left(z_{1}^{2}+z_{2}^{2}\right)+e_{2}\left(z_{1}^{2}-z_{2}^{2}\right)+e_{3}\left(2 z_{1} z_{2}\right) .
$$

Let $P_{1}, P_{2}$ and $P_{3}$ denote the natural $\mathbf{Q}_{8}$-projections onto the subspaces of $\operatorname{Sym}_{\mathbb{C}}^{2}(\mathbb{H})$ spanned by $\left\{e_{1}, e_{2}\right\},\left\{e_{2}, e_{3}\right\}$ and $\left\{e_{1}, e_{3}\right\}$, respectively. A direct calculation shows that $\operatorname{ker} P_{i} \cap \triangle(\mathbb{H})=\{0\}$ for $i=1,2,3$. Consequently $f_{i}=P_{i} \circ \triangle, i=1,2,3$, are $\mathbf{Q}_{8}$-equivariant nonsingular maps. Notice that the map $f_{1}$ is exactly the map $\tilde{f}$ from Example 2.1 .

Remark 3.4. Observe that in Example 3.3 the coordinates of the "diagonal" map are complex homogeneous polynomials. It follows immediately from the Hilbert Nullstellen Theorem that it is impossible to construct, using complex symmetric powers, an equivariant nonsingular map $f: V \rightarrow W$ 
decreasing dimension (i.e. $\operatorname{dim} V>\operatorname{dim} W$ ). However, as it is shown in Examples 2.5 and 2.8 and Remark 2.9, there are equivariant nonsingular maps decreasing dimension with coordinates given by real homogeneous polynomials. This indicates the use of real symmetric powers for the construction of equivariant maps decreasing dimension.

Example 3.5. The quaternionic algebra $\mathbb{H}$ is a 4 -dimensional real vector space where a vector $h=z_{1}+j z_{2}, z_{1}=x_{1}+j y_{1}$ and $z_{2}=x_{2}+j y_{2}$, can be represented as the linear combination $h=1 \cdot x_{1}+i \cdot y_{1}+j \cdot x_{2}+j i \cdot y_{2}$. Therefore, the second real symmetric power $\operatorname{Sym}_{\mathbb{R}}^{2}(\mathbb{H})$ admits the following invariant basis:

$$
\begin{array}{ll}
e_{1}=\frac{1 \otimes 1-j \otimes j-i \otimes i+j i \otimes j i}{4}, & e_{2}=\frac{1 \otimes i+i \otimes 1+j \otimes j i+j i \otimes j}{4} \\
e_{3}=\frac{1 \otimes j+j \otimes 1-i \otimes j i-j i \otimes i}{4}, & e_{4}=\frac{i \otimes j+j \otimes i+1 \otimes j i+j i \otimes 1}{4}, \\
e_{5}=\frac{1 \otimes 1+j \otimes j-i \otimes i-j i \otimes j i}{4}, & e_{6}=\frac{1 \otimes i+i \otimes 1-j \otimes j i-j i \otimes j}{4} \\
e_{7}=\frac{1 \otimes j+j \otimes 1+i \otimes j i+j i \otimes i}{4}, & e_{8}=\frac{i \otimes j+j \otimes i-1 \otimes j i-j i \otimes 1}{4} \\
e_{9}=\frac{1 \otimes 1-j \otimes j+i \otimes i-j i \otimes j i}{4}, & e_{10}=\frac{1 \otimes 1+j \otimes j+i \otimes i+j i \otimes j i}{4},
\end{array}
$$

and the "diagonal" map $\triangle: \mathbb{H} \rightarrow \operatorname{Sym}_{\mathbb{R}}^{2}(\mathbb{H})$ in the above basis $\left\{e_{1}, e_{2}, e_{3}, e_{4}\right.$, $\left.e_{5}, e_{6}, e_{7}, e_{8}, e_{9}, e_{10}\right\}$ is given by

$$
\begin{aligned}
\triangle\left(z_{1}+j z_{2}\right)= & \left(x_{1}^{2}-x_{2}^{2}-y_{1}^{2}+y_{2}^{2}, 2\left(x_{1} y_{1}+x_{2} y_{2}\right), 2\left(x_{1} x_{2}-y_{1} y_{2}\right),\right. \\
& 2\left(x_{2} y_{1}+x_{1} y_{2}\right), \\
& x_{1}^{2}+x_{2}^{2}-y_{1}^{2}-y_{2}^{2}, 2\left(x_{1} y_{1}-x_{2} y_{2}\right), 2\left(x_{1} x_{2}+y_{1} y_{2}\right), \\
& 2\left(x_{2} y_{1}-x_{1} y_{2}\right), \\
& \left.x_{1}^{2}-x_{2}^{2}+y_{1}^{2}-y_{2}^{2}, x_{1}^{2}+x_{2}^{2}+y_{1}^{2}+y_{2}^{2}\right) \\
= & \left(z_{1}^{2}-\bar{z}_{2}^{2}, 2 z_{1} z_{2}, z_{1}^{2}+\bar{z}_{2}^{2}, 2 z_{1} \bar{z}_{2},\left|z_{1}\right|^{2}-\left|z_{2}\right|^{2},\left|z_{1}\right|^{2}+\left|z_{2}\right|^{2}\right) .
\end{aligned}
$$

Notice that if we change the order of the basis to $\left\{e_{5}, e_{2}, e_{1}, e_{6}, e_{7}, e_{8}, e_{9}, e_{3}, e_{4}\right.$, $\left.e_{10}\right\}$ then the map $\triangle$ can be written as follows:

$$
\triangle\left(z_{1}+j z_{2}\right)=\left(z_{1}^{2}+z_{2}^{2}, z_{1}^{2}-z_{2}^{2}, 2 z_{1} \bar{z}_{2},\left|z_{1}\right|^{2}-\left|z_{2}\right|^{2}, 2 z_{1} z_{2},\left|z_{1}\right|^{2}+\left|z_{2}\right|^{2}\right) .
$$

Let $P_{1}$ and $P_{2}$ be the natural projections on the subspaces spanned by $\left\{e_{1}, e_{2}, e_{3}\right\}$ and $\left\{e_{1}, e_{6}, e_{7}\right\}$, respectively. By direct verification one can easily check that $\operatorname{ker} P_{i} \cap \triangle(\mathbb{H})=\{0\}, i=1,2$, and therefore, $f_{i}=P_{i} \circ \triangle$, $i=1,2$, are nonsingular $\mathbf{Q}_{8}$-equivariant maps which were presented in $(2.2)$ and (2.3), respectively.

Remark 3.6. Notice that, if $f: V \rightarrow W$ is a nonsingular $G$-equivariant map with coordinates being homogeneous polynomials of degree $k$, then there exists a $G$-equivariant linear operator $T: W \rightarrow \operatorname{Sym}^{k}(V)$ such that 
$T \circ f=\triangle$. In this sense the above construction of "homogeneous" nonsingular equivariant maps is generic. Clearly, it is possible to combine different symmetric powers in order to construct more complicated examples of equivariant maps (see [6], Example 4.1).

Practical applications of the construction described above are related to several classical problems of the representation theory:

(i) decomposition of symmetric power $\operatorname{Sym}^{k}(V)$ into irreducible components (spectral decomposition);

(ii) finding invariant bases in $\operatorname{Sym}^{k}(V)$ (Clebsch-Gardon problem);

(iii) verification of the condition (A) (a sort of an equivariant transversality).

There is a vast literature devoted to the problems (i) and (ii) for the classical groups (see [4] and [13] and the references therein). It is our intention to elaborate the above construction for certain important representations of classical (infinite) groups (for example, $S U(n)$ and $\operatorname{Sp}(n)$ ). Observe also that Remarks 2.6 and 2.9 give rise to the question if there is any congruence relation, similar to (1.1), for Hopf invariants of equivariant maps from $S^{4 n-1}$ to $S^{2 n}$ (see also $\left.[3,5,6]\right)$.

\section{Applications}

Let $f: \mathbb{R}^{n} \rightarrow \mathbb{R}^{n}$ and $g:[0, \omega] \times \mathbb{R}^{n} \rightarrow \mathbb{R}^{n}$ be two maps. We are making the following assumptions:

(H1) $f$ is a positively homogeneous map with constant $\alpha \geq 1$, i.e. $f(t v)=$ $t^{\alpha} f(v)$ for every $t>0$ and $v \in \mathbb{R}^{n}$

(H2) $g$ satisfies the Carathéodory conditions;

(H3) $v \equiv 0$ is the only bounded solution of $\dot{v}=f(v)$;

(H4) $\lim _{\|v\| \rightarrow \infty}\|g(t, v)\| /\|v\|^{\alpha}=0$ uniformly a.e. in $t \in[0, \omega]$;

(H5) $\operatorname{deg}(f, B(0, \varepsilon)) \neq 0$ for some $\varepsilon>0$, where $B(0, \varepsilon)=\left\{v \in \mathbb{R}^{n} ;\|v\|<\varepsilon\right\}$. We consider the following system of ODEs:

$$
\dot{v}=f(v)+g(t, v), \quad v \in \mathbb{R}^{n} .
$$

The systems of type (4.1), i.e. perturbation of autonomous systems with positively homogeneous nonlinearity, were widely studied (see [7,11] and references therein).

Proposition 4.1. (cf. [11], [12], Cor. 4.7). Assume that $f: \mathbb{R}^{n} \rightarrow \mathbb{R}^{n}$ and $g:[0, \omega] \times \mathbb{R}^{n} \rightarrow \mathbb{R}^{n}$ satisfy the assumptions (H1) - (H5). Then (4.1) has at least one $\omega$-periodic solution.

As an immediate consequence of Propositions 4.1 and 2.2 we obtain

Theorem 4.2. Let $f: \mathbb{H} \rightarrow \mathbb{C}^{2}$ and $g:[0, \omega] \times \mathbb{R}^{4} \rightarrow \mathbb{R}^{4}$ satisfy assumptions (H1)-(H4). If $f$ is a nonsingular $\mathbf{Q}_{8}$-equivariant map, then (4.1) has at least one $\omega$-periodic solution. 
Example 4.3. Take $f: \mathbb{H} \rightarrow \mathbb{C}^{2}, f\left(z_{1}+j z_{2}\right)=\left(\bar{z}_{1}^{2}+\bar{z}_{2}^{2}, \bar{z}_{1}^{2}-\bar{z}_{2}^{2}\right),\left(z_{1}, z_{2}\right) \in$ $\mathbb{C}^{2}=\mathbb{R}^{4}$. Clearly, $f$ is a nonsingular $\mathbf{Q}_{8}$-equivariant map. Assume that $g:[0, \omega] \times \mathbb{R}^{4} \rightarrow \mathbb{R}^{4}$ satisfies conditions (H2) and (H4) with $\alpha=2$. Then system (4.1) has at least one $\omega$-periodic solution. Indeed, we only need to show that the system $\dot{v}=f(v), v \in \mathbb{R}^{4}$, does not have any bounded solution except $v \equiv 0$. Indeed, assume that $\nu(t)=\left(z_{1}(t), z_{2}(t)\right)$ is a non-zero bounded solution of $\dot{v}=f(v)$ and put $\varphi(t):=\Phi(\nu(t))$, where $\Phi\left(z_{1}, z_{2}\right)=\operatorname{Re}\left(z_{1}^{3}-\right.$ $\left.z_{2}^{3}\right)$. Notice that $\varphi^{\prime}(t)=<\nabla \Phi\left(z_{1}(t), z_{2}(t)\right), \dot{\nu}(t)>=3\left(\left|z_{1}(t)\right|^{4}+\left|z_{2}(t)\right|^{4}\right)$, thus the function $\varphi(t)$ is strictly increasing and bounded. In particular, $\lim _{t \rightarrow \pm \infty} \varphi(t)=a_{ \pm}\left(\left|a_{ \pm}\right|<\infty\right)$ and hence $\lim _{t \rightarrow \pm \infty} \varphi^{\prime}(t)=0$. Since $\varphi^{\prime}(t)=$ $3\left(\left|z_{1}(t)\right|^{4}+\left|z_{2}(t)\right|^{4}\right)$, it follows that $\lim _{t \rightarrow \pm \infty} \nu(t)=0$, but this contradicts $\varphi(t)$ is a strictly increasing function.

Remark 4.4. Consider the map $\psi\left(z_{1}, z_{2}\right)=\left(\bar{z}_{1}^{2 n}+\bar{z}_{2}^{2 n}, \bar{z}_{1}^{2 n}-\bar{z}_{2}^{2 n}\right)$, where $n \geq 1$ is an odd integer. It is clear that $\psi$ is $\mathbf{Q}_{8}$-equivariant, positively homogeneous and nonsingular. It may be verified that it also satisfies the assumption (H3).

As another application we consider the following boundary value problem

$$
\left\{\begin{array}{l}
x^{(n)}=F\left(x, x^{\prime}, \ldots, x^{(n-1)}, t\right), \\
x^{(i)}(0)=x^{(i)}(\omega), \quad i=0,1, \ldots, n-1,
\end{array}\right.
$$

where $x \in \mathbb{R}^{4}, F: \mathbb{R}^{4 n} \times[0,1] \rightarrow \mathbb{R}^{4}$ is continuously differentiable and $\omega \in(0,1)$ but is not specified. Similar problems, in more general setting (cf. [10] and the references therein) were considered by many authors. By the well known result of K. Schmitt (cf. [10], Theorem 9.4.1) and Proposition 2.2 we immediately obtain

Theorem 4.5. Let $D \subset \mathbb{H}$ be an open bounded set containing zero and $h: \mathbb{H} \rightarrow \mathbb{H}$ be defined by $h(x)=F(x, 0, \ldots, 0)$. If $h$ is homotopic to a $\mathbf{Q}_{8}$-equivariant nonsingular map $f:\left(\mathbb{H}, \mathbf{Q}_{8}\right) \rightarrow\left(\mathbb{C}^{2}, \mathbf{Q}_{8} / \mathbb{Z}_{2}\right)$, by a homotopy without zeros on $\partial D$, then there exists $\omega_{0}>0$ such that (4.2) has a solution for each $\omega \in\left(0, \omega_{0}\right)$.

\section{REFERENCES}

[1] J. F. Adams, Seattle Conference on Differential and Algebraic Topology, Collection of Unsolved Problems, 1963.

[2] M. F. Atiyah and D. O. Tall, Group representations, $\lambda$-rings and $J$-homomorphisms, Topology 8 (1969), 253-297.

[3] T. Bartsch, Topological Methods for Variational Problems with Symmetries, Lect. Notes in Math. 1560, Springer, Berlin, 1993.

[4] W. Fulton and J. Harris, Representation Theory. A First Course, Graduate Texts in Math. 129, Springer, New York, 1991.

[5] J. Ize and A. Vignoli, Equivariant degree for abelian actions, Part I: Equivariant homotopy groups, Topol. Mathods Nonl. Anal. 2 (1993), 367-413.

[6] J. Ize and A. Vignoli, Equivariant degree for abelian actions, Part II: Index Computations, Topol. Methods Nonl. Anal. 7 (1996), 369-430. 
[7] M. Krasnosel'skii and P. Zabreiko, Geometrical Methods of Nonlinear Analysis, Springer, New York, 1984.

[8] A. Kushkuley and Z. Balanov, Geometric Methods in Degree Theory for Equivariant Maps, Lect. Notes in Math. 1632, Springer, Berlin, 1996.

[9] C. N. Lee and A. Wasserman, On the groups $J O(G)$.' Mem. Amer. Math. Soc. 159, Providence, Rhode Island, 1975.

[10] N. G. Lloyd, Degree Theory, Cambridge Tracks in Mathematics, 73, Cambridge Univ. Press, London, 1978.

[11] J. Mawhin, Topological degree and boundary value problems for nonlinear differential equations', in Topological Methods for Ordinary Differential Equations, (M. Furi and P. Zecca eds), Lect. Notes in Math. 1537, Springer, New York, 1993, 74-142.

[12] E. Muhamadiev, Construction of a correct guiding function for a system of differential equations, Soviet Math. Dokl. 11 (1970), 202-205.

[13] N. Ja. Vilenkin and A.U. Klimyk, Representation of Lie Groups and Special Functions. Recent Advances, Kluwer, London, 1995.

ZALMAN BALANOV

Department of Mathematics and Computer Science

BAR ILAN UNIVERSITY

52900 RAMAT-GAN, ISRAEL

Current address:

Department of Mathematical Sciences

UNIVERSITY OF ALBERTA

Edmonton, Alberta

T6G 2G1 CANADA

WIESLAW KRAWCEWICZ

Department of Mathematical Sciences

University of Alberta

Edmonton, Alberta

T6G 2G1 CANADA

Alexander Kushiuley

6 Carriage Drive

ACTON, MA 01720, USA 


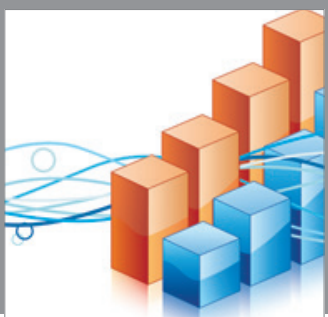

Advances in

Operations Research

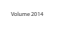

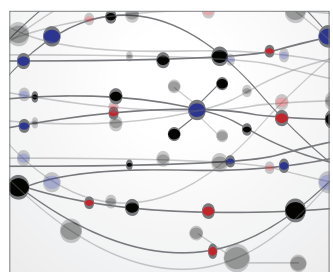

\section{The Scientific} World Journal
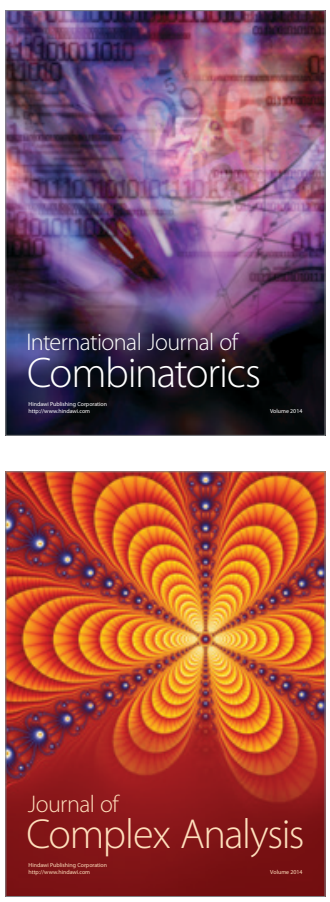

International Journal of

Mathematics and

Mathematical

Sciences
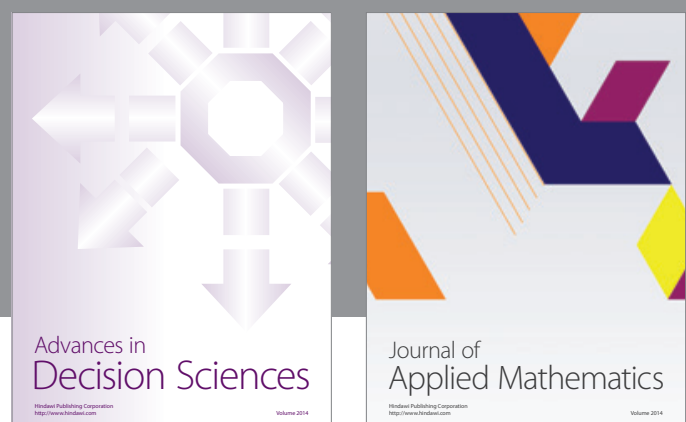

Journal of

Applied Mathematics
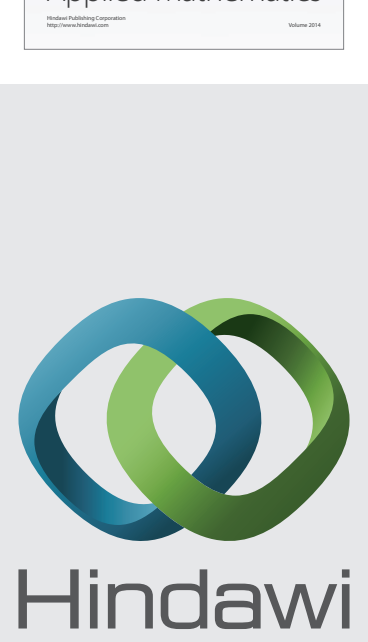

Submit your manuscripts at http://www.hindawi.com
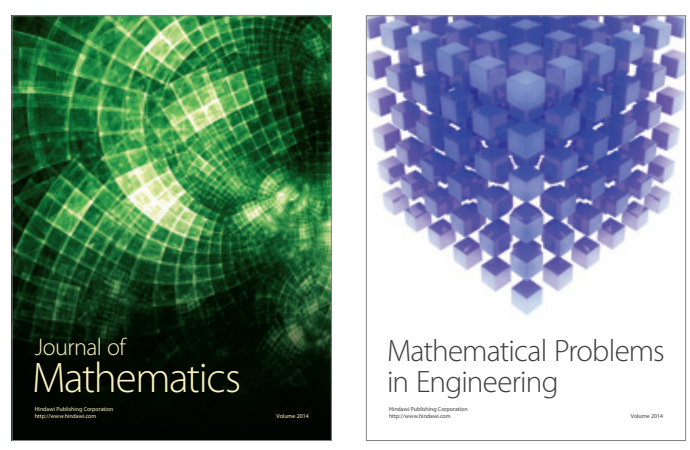

Mathematical Problems in Engineering
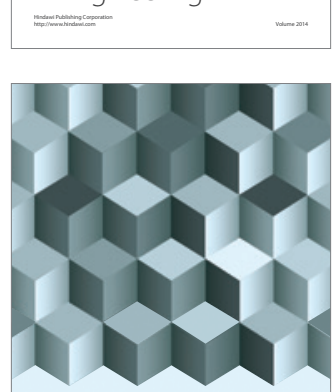

Journal of

Function Spaces
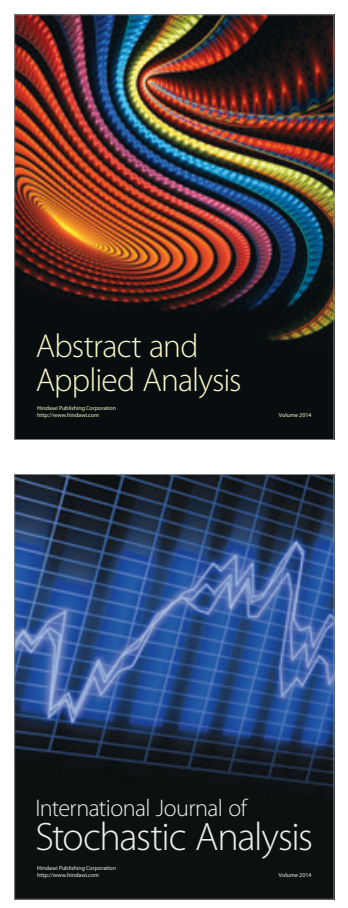

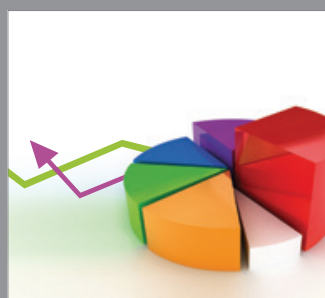

ournal of

Probability and Statistics

Promensencen
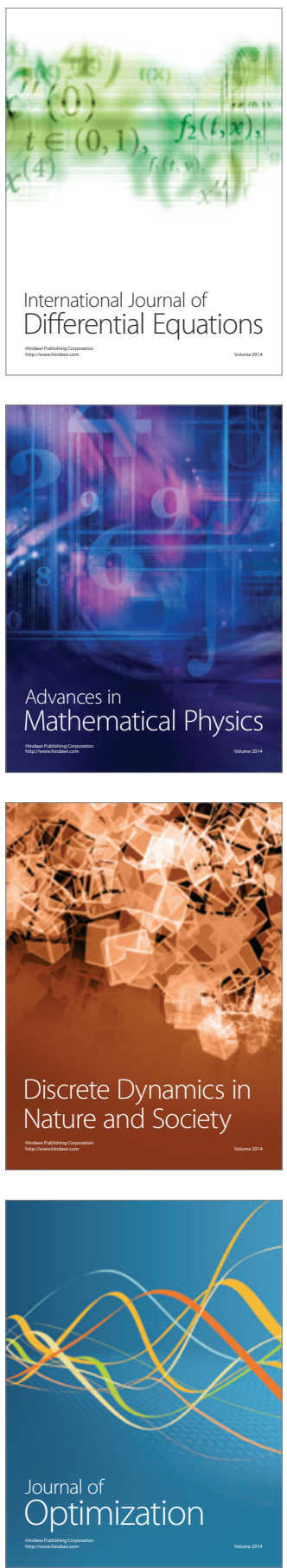